\title{
Skeletal myoblast sheet transplantation improves the diastolic function of a pressure-overloaded right heart
}

\author{
Takaya Hoashi, MD, ${ }^{\mathrm{a}}$ Goro Matsumiya, $\mathrm{MD}, \mathrm{PhD},{ }^{\mathrm{a}}$ Shigeru Miyagawa, $\mathrm{MD}, \mathrm{PhD},{ }^{\mathrm{a}}$ \\ Hajime Ichikawa, MD, PhD, ${ }^{a}$ Takayoshi Ueno, $\mathrm{MD}, \mathrm{PhD},{ }^{\mathrm{a}}$ Masamichi Ono, $\mathrm{MD}, \mathrm{PhD},{ }^{\mathrm{a}}$ \\ Atsuhiro Saito, $\mathrm{PhD},{ }^{\mathrm{a}}$ Tatsuya Shimizu, MD, PhD, ${ }^{\mathrm{b}}$ Teruo Okano, MD, PhD, ${ }^{\mathrm{b}}$ Naomasa Kawaguchi, $\mathrm{PhD},{ }^{\mathrm{c}}$ \\ Nariaki Matsuura, $\mathrm{MD}, \mathrm{PhD},{ }^{\mathrm{c}}$ and Yoshiki Sawa, $\mathrm{MD}, \mathrm{PhD}^{\mathrm{a}}$
}

\begin{abstract}
Objective: The development of right ventricular dysfunction has become a common problem after surgical repair of complex congenital heart disease. A recent study reported that tissue-engineered skeletal myoblast sheet transplantation improves left ventricular function in patients with dilated and ischemic cardiomyopathy. Therefore myoblast sheet transplantation might also improve ventricular performance in a rat model of a pressure-overloaded right ventricle.
\end{abstract}

\begin{abstract}
Methods: Seven-week-old male Lewis rats underwent pulmonary artery banding. Four weeks after pulmonary artery banding, myoblast sheet transplantation to the right ventricle was performed in the myoblast sheet transplantation group $(n=20)$, whereas a sham operation was performed in the sham group $(n=20)$.
\end{abstract}

\begin{abstract}
Results: Four weeks after performing the procedure, a hemodynamic assessment with a pressure-volume loop showed a compensatory increase in systolic function in both groups. However, only the myoblast sheet transplantation group showed a significant improvement in the diastolic function: end-diastolic pressure (sham vs myoblast sheet transplantation, $10.3 \pm 3.1$ vs $5.0 \pm 3.7 \mathrm{~mm} \mathrm{Hg} ; P<.001)$, time constant of isovolumic relaxation $(11.1 \pm$ 2.5 vs $7.6 \pm 1.2 \mathrm{~ms}, P<.001)$, and end-diastolic pressure-volume relationship $(16.1 \pm 4.5 \mathrm{vs} 7.6 \pm 2.4 / \mathrm{mL}$, $P<.005)$. The right ventricular weight and cell size similarly increased in both groups. A histologic assessment demonstrated significantly suppressed ventricular fibrosis and increased capillary density in the myoblast sheet transplantation group in comparison with those in the sham group. Reverse transcription-polymerase chain reaction demonstrated an increased myocardial gene expression of hepatocyte growth factor and vascular endothelial growth factor in the myoblast sheet transplantation group but not in the sham group.
\end{abstract}

Conclusions: Skeletal myoblast sheet transplantation improved the diastolic dysfunction and suppressed ventricular fibrosis with increased capillary density in a rat model of a pressure-overloaded right ventricle. This method might become a novel strategy for the myocardial regeneration of right ventricular failure in patients with congenital heart disease.

Because of recent developments in diagnostic methods, the establishment of new surgical techniques, and improvements in perioperative management, patients with complex congenital heart disease (CHD) are today often able to survive to adulthood. However, even after a successful repair, right ventricular (RV) overload remains in some patients, in whom it impairs RV function and influences long-term mortality and morbidity. ${ }^{1-3}$ Chronic pressure overload is one of the major risk factors of RV dysfunction. In this

From the Department of Cardiovascular Surgery, ${ }^{\text {a }}$ Osaka University Graduate School of Medicine, Osaka, Japan; the Institute of Advanced Biomedical Engineering and

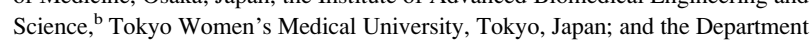
of Molecular Pathology, ${ }^{\mathrm{c}}$ Osaka University Graduate School of Allied Health Science, Osaka, Japan.

Received for publication March 17, 2008; revisions received Oct 1, 2008; accepted for publication Feb 2, 2009.

Address for reprints: Yoshiki Sawa, MD, PhD, 2-2 Yamadaoka, Suita, Osaka 565-

0871, Japan (E-mail: sawa@surg1.med.osaka-u.ac.jp).

J Thorac Cardiovasc Surg 2009;138:460-7

$0022-5223 / \$ 36.00$

Copyright $@ 2009$ by The American Association for Thoracic Surgery

doi:10.1016/j.jtcvs.2009.02.018 situation the right ventricle is hypertrophied and systolic function is initially preserved, whereas diastolic function gradually deteriorates. ${ }^{4,5}$ Prolonged exposure to excessive pressure overload results in irreversible RV failure. Clinically, the relationship between progressive fibrosis and RV function must be addressed. ${ }^{6-8}$

Recently, cardiac regeneration therapy has provided a new treatment for end-stage heart failure, and skeletal myoblasts are currently thought to be a potential cell source. ${ }^{9-11} \mathrm{We}$ developed a novel cell delivery system using temperatureresponsive culture dishes, ${ }^{12}$ and tissue-engineered cell sheets have been created without any scaffold, which maintains cellcell interaction and extracellular matrix while avoiding any inflammatory reaction, and with improved cell survival. ${ }^{13}$ Skeletal myoblast sheet transplantation (MST) has been shown to improve left ventricular (LV) contractility in several animal models of LV failure. ${ }^{14-16}$ Otherwise, it is unclear whether MST can also affect the right ventricle, especially pressure-induced RV dysfunction. Hence this study assessed whether MST could improve RV function in rats after damage caused by pressure overload. 


\begin{tabular}{|c|c|}
\hline \multicolumn{2}{|c|}{ Abbreviations and Acronyms } \\
\hline BW & $=$ body weight \\
\hline CFR & $=$ coronary flow reserve \\
\hline EDPVR & $\begin{aligned}= & \text { end-diastolic pressure-volume } \\
& \text { relationship }\end{aligned}$ \\
\hline Ees & $=$ end-systolic elastance \\
\hline ESPVR & $\begin{aligned}= & \text { end-systolic pressure-volume } \\
& \text { relationship }\end{aligned}$ \\
\hline GAPDH & $\begin{aligned} I= & \text { glyceraldehyde- } 3 \text {-phosphate } \\
& \text { dehydrogenase }\end{aligned}$ \\
\hline HGF & $=$ hepatocyte growth factor \\
\hline IVS & $=$ intraventricular septum \\
\hline LV & $=$ left ventricular \\
\hline MS & $=$ myoblast cell sheet \\
\hline MST & $=$ myoblast sheet transplantation \\
\hline PA & $=$ pulmonary artery \\
\hline PAB & $=$ pulmonary artery banding \\
\hline PRSW & $=$ preload recruitable stroke work \\
\hline RT-PCR & $\begin{aligned}= & \text { reverse transcription-polymerase } \\
& \text { chain reaction }\end{aligned}$ \\
\hline RV & $=$ right ventricular \\
\hline SW & $=$ stroke work \\
\hline VEGF & $=$ vascular endothelial growth factor \\
\hline
\end{tabular}

\section{MATERIALS AND METHODS}

\section{Animal Care}

All experimental procedures and protocols used in this investigation were reviewed and approved by the institutional animal care and use committee and are in accordance with the National Institutes of Health "Guide for the care and use of laboratory animals" (National Institutes of Health publication no. 85-23, revised 1996).

\section{Creation of Chronic RV Pressure Overload}

A rat model of pulmonary artery banding (PAB) was established to create chronic RV pressure overload. Seven-week-old male Lewis rats (180-210 g) were anesthetized with an intraperitoneal injection of ketamine hydrochloride $(50 \mathrm{mg} / \mathrm{kg})$ and xylazine $(5 \mathrm{mg} / \mathrm{kg})$ and ventilated by using a volumecontrolled respirator ( $2 \mathrm{~mL}, 60$ cycles/min) with room air. A left thoracotomy was performed at the fourth intracostal space, and the main pulmonary artery (PA) was carefully exposed. As previously reported, ${ }^{17}$ a 19 -gauge injection needle (outer diameter, $1.1 \mathrm{~mm}$ ) was placed alongside the PA, and a 3-0 polyester suture was tied tightly around the PA and the needle. Next, the needle was rapidly removed, and then a fixed diameter was set for the PA. Thereafter, the thorax was closed in layers, and the ventilator setting was changed (90 cycles/min) for half an hour to reduce the respiratory load.

\section{Skeletal Myoblast Sheet Preparation}

Creation of myoblast cell sheets (MSs) with temperature-responsive culture dishes (UpCell; Cellseed, Tokyo, Japan) was done according to previous reports. ${ }^{14-16}$ Briefly, skeletal muscle was harvested from the hind legs of 4-week-old syngeneic rats. The purified myoblasts were incubated on 35 -mm UpCell dishes at $37^{\circ} \mathrm{C}$, with the cell numbers adjusted to $3 \times 10^{6}$ per dish. After 12 to 18 hours, the dishes were moved to a refrigerator set at $20^{\circ} \mathrm{C}$ and left there for 30 minutes. During that time, the MSs detached spontaneously from the surfaces. Each sheet measured from 10 to $15 \mathrm{~mm}$ in diameter.

\section{Skeletal MST}

Four weeks after PAB, a second left thoracotomy was performed at the fifth intracostal space after achievement of general anesthesia. After opening the pericardium, the RV anterior wall was exposed. Two MSs were grafted onto each anterior wall of the right ventricle in the MST group $(n=20)$, or a sham operation was performed in the sham group $(n=20)$. The pericardium was closed linearly before the thorax was closed to prevent the dislocation of MSs. In addition, age-matched rats that did not undergo surgical intervention were also prepared as a control group $(n=20)$.

\section{Hemodynamic Study and Data Analysis}

Four weeks after the MST or sham operation, 10 rats in each group were anesthetized and ventilated again and were set on the blanket warmer to maintain body temperature. A median sternotomy was performed, and the pericardium was opened carefully to minimize hemorrhaging. A silk thread was placed under the inferior vena cava just above the diaphragm to change the RV preload. After purse-string sutures were attached with 7-0 polypropylene, the conductance catheter (Unique Medical Co, Tokyo, Japan) was inserted through the RV apex toward the pulmonary valve along the longitudinal axis of the RV cavity and then fixed. A Miller 1.4F pressure-tip catheter (SPR-719; Millar Instruments, Houston, Tex) was also inserted from the RV anterior wall and fixed. For better volume measurement, a 1-mm curve was added to the original standard straight conductance catheter to fit the complex RV geometry. The position of the conductance catheter was determined by observing the pressure and segmental volume signals with the appropriate phase relationships. The conductance system and the pressure transducer controller (Integral 3 [VPR-1002], Unique Medical Co) were set as previously reported. ${ }^{18}$ Pressure-volume loops and intracardiac electrocardiograms were monitored online, and the conductance, pressure, and intracardiac electrocardiographic signals were analyzed with Integral 3 software (Unique Medical Co). ${ }^{18}$

Under stable hemodynamic conditions, the baseline indices were initially measured, and then the pressure-volume loop was drawn during inferior vena caval occlusion and analyzed (Figure 1). Finally, the conductivity of the sampled blood was measured with a small $(0.1 \mathrm{~mm})$ cuvette, and the parallel conductance volume was measured with the hypertonic saline dilution method to obtain the absolute volumes. ${ }^{19}$

The following indices were calculated as the baseline RV function: heart rate, end-systolic pressure, end-diastolic pressure, $\mathrm{dP} / \mathrm{dtmax}, \mathrm{dP} / \mathrm{dtmin}$, and the time constant of isovolumic relaxation $(\tau)$. The following relationships were determined by means of pressure-volume loop analysis as load-independent measures of RV function: end-systolic pressure-volume relationship (ESPVR), end-diastolic pressure-volume relationship (EDPVR), and preload recruitable stroke work (PRSW).

The ESPVR is linear, and it can be characterized by a slope (endsystolic elastance [Ees]) and a volume axis intercept (Vo), so that Pes $=$ Ees $(V e s-V o)$, where Pes and Ves are the end-systolic pressure and volume, respectively. ${ }^{20}$

In contrast, the EDPVR is intrinsically thought to be nonlinear. The relationship between the end-diastolic pressure (Ped) and volume (Ved) can be fitted to the monoexponential, so that $P e d=P o+b e^{K v V e d}$, where Po is the pressure asymptote (generally close to $0 \mathrm{~mm} \mathrm{Hg}$ ), b is a constant, and $\mathrm{Kv}$ is the variable represented as a ventricular stiffness property. ${ }^{21}$

The relationship between ventricular stroke work (SW) and end-diastolic volume (Ved) is represented as PRSW. PRSW is thought to be a suitable parameter of the contractile state and fitted to the following equation: $S W=K(V e d-V o)$, where $\mathrm{K}$ is a constant as a potential measure of intrinsic myocardial performance independent of loading, geometry, and heart rate. ${ }^{22}$

\section{Histopathologic Analysis}

The other 10 rats in each group were killed 4 weeks after the sham or MST operation for histologic analysis, reverse transcription-polymerase chain reaction (RT-PCR), and blood sampling. The hearts were quickly removed, and the ventricles were dissected free of atrial tissue and large 
Control

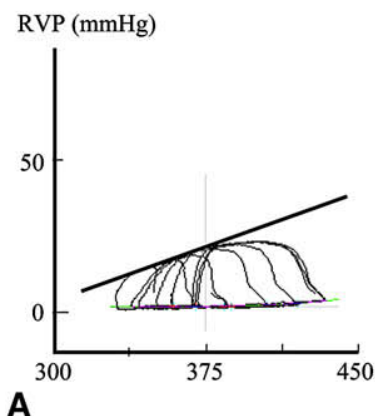

Sham

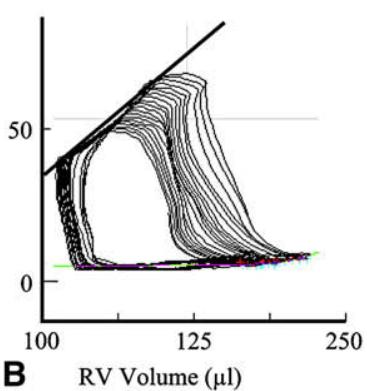

MST

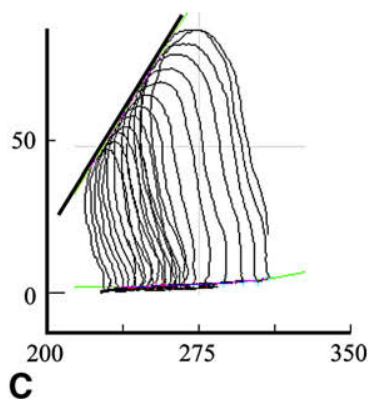

FIGURE 1. Representative pressure-volume loops of the control (A), sham (B), and myoblast sheet transplantation (MST; C) groups under different loading conditions. The slope of the end-systolic pressure-volume relationship is displayed as a black straight line. The correlation of the end-diastolic pressurevolume relationship is displayed as a green monoexponential curve. $R V P$, Right ventricular pressure; $R V$, right ventricle.

blood vessels. The right ventricle was carefully separated from the left ventricle and intraventricular septum (IVS). The fresh ventricular tissues were immediately blotted dry and weighted separately to determine the degree of RV hypertrophy based on 2 parameters: RV wall weight/body weight $(\mathrm{RV} / \mathrm{BW})$ and $\mathrm{RV}$ wall weight/LV and IVS wall weight (RV/ LVHVS).

Tissue specimens were obtained from the endocardium, the midwall, and the epicardium of the RV anterior wall in cross-sections, cut into $5-\mu \mathrm{m}$-thick sections, and stained with hematoxylin and eosin for morphologic analysis, including measurement of RV wall thickness, periodic acidSchiff staining to measure the short-axis length of the RV myocardial cell, Factor VIII-related antigen staining (Dako EPOS anti-human Von Willebrand factor/HRP; Dako Cytomation, Glostrup, Denmark) to quantify capillary vascular density, and Masson trichrome staining for determination of the amount of interstitial and myocardial fibrosis. The percentage of interstitial and myocardial fibrosis were assessed by a computer-based method $^{23,24}$ with the use of a software filter (Mac Scope Software; MITANI Corp, Tokyo, Japan), which can recognize the distinct color shades. The number of pixels of the blue-stained collagen area was calculated, then divided by the total number of pixels in a field. Each 3 fields of the endocardial, epicardial, and mid layers of the RV wall per slide were analyzed and then averaged.

\section{RT-PCR}

Total RNA was isolated from the stored specimens by using the RNeasy Mini Kit (Qiagen, Hilden, Germany) and reverse transcribed with Omniscript Reverse Transcriptase (Qiagen). RT-PCR was performed with the ABI PRISM 7700 (Applied Biosystems, Foster City, Calif). Measurement of the mRNA expression of hepatocyte growth factor (HGF) and vascular endothelial growth factor (VEGF) was performed in triplicate. The results are expressed after normalization for glyceraldehyde-3-phosphate dehydrogenase (GAPDH).

\section{Statistical Analysis}

All data were expressed as the mean \pm SEM and range. Student's unpaired $t$ test or analysis of variance for parametric values was used to compare group means.

\section{RESULTS}

\section{Pressure Overload and Hypertrophy of the Right Ventricle After PAB}

After PAB, a weight analysis showed the heart weight/BW, $\mathrm{RV} / \mathrm{BW}$, and $\mathrm{RV} /(\mathrm{LV}+\mathrm{IVS})$ weight ratios in the sham and
MST groups to be similar and significantly higher than in the control group (Table 1). Both the sham and MST groups showed a significantly increased end-systolic pressure and $\mathrm{dP} / \mathrm{dtmax}$ than seen in the control group (Table 2).

\section{Hemodynamic Effects of MST}

The baseline indices revealed that end-diastolic pressure and $\tau$ values were significantly increased only in the sham group in comparison with those in the control group but not in the MST group (Table 2). Typical examples of the pressure-volume loop in each group are presented in Figure 1 . The pressure-volume loop analysis revealed that the ESPVR and PRSW values significantly increased both in the sham and MST groups. However, the EDPVR value significantly increased only in the sham group (control vs sham vs MST groups: $8.6 \pm 2.9$ vs $16.1 \pm 4.5$ vs $7.6 \pm$ $2.4 / \mathrm{mL} ; P<.05$ in the control and MST groups vs the sham group; Table 2).

\section{Histologic Effects of MST}

Whole heart findings showed the RV wall thickened, the cavity enlarged, and the IVS shifted toward the left side in the sham and MST groups (Figure 2, A). In the MST group transplanted MSs were observed as an elastic thin layer on the epicardium (Figure 2, B). The RV wall thickness and myocardial cell size in the sham and MST groups were similar and significantly higher than in the control group (Figure 2, $C-E$ ).

TABLE 1. Weight analysis at the fourth week after the operation

\begin{tabular}{lccc}
\hline \multicolumn{1}{c}{ Group } & Control & Sham & MST \\
\hline HW/BW (mg/g) & $2.62 \pm 0.09$ & $3.53 \pm 0.50^{*}$ & $4.03 \pm 0.59^{*}$ \\
RV/BW (mg/g) & $0.54 \pm 0.15$ & $1.62 \pm 0.42^{*}$ & $1.65 \pm 0.32^{*}$ \\
RV/(IVS HL) & $0.27 \pm 0.08$ & $0.69 \pm 0.11^{*}$ & $0.69 \pm 0.09 *$ \\
\hline
\end{tabular}

$M S T$, Myoblast sheet transplantation; $H W$, heart weight; $B W$, body weight; $R V$, right ventricle; IVS, interventricular septum; $L V$, left ventricle. ${ }^{*} P<.05$ versus the control group. 
TABLE 2. Hemodynamic indices at the fourth week after the operation

\begin{tabular}{lccc}
\hline \multicolumn{1}{c}{ Group } & Control & Sham & MST \\
\hline Basic hemodynamic indices & & & \\
HR (beats/min) & $280 \pm 72$ & $233 \pm 34$ & $249 \pm 65$ \\
ESP (mm Hg) & $22.8 \pm 2.9$ & $82.3 \pm 11.8^{*}$ & $78.7 \pm 13.2^{*}$ \\
EDP (mm Hg) & $2.4 \pm 1.4$ & $10.3 \pm 3.1^{*} \dagger$ & $5.0 \pm 3.7$ \\
dP/dtmax (mm Hg/s) & $1301 \pm 206$ & $3197 \pm 597^{*}$ & $3352 \pm 1332^{*}$ \\
dP/dtmin (mm Hg/s) & $-997 \pm 210$ & $-2466 \pm 582^{*}$ & $-2682 \pm 828^{*}$ \\
$\tau(\mathrm{ms})$ & $7.9 \pm 2.7$ & $11.1 \pm 2.5^{*} \dagger$ & $7.6 \pm 1.2$ \\
Load-independent parameters analyzed by PV loop & & & \\
ESPVR (mm Hg/mL) & $538 \pm 196$ & $857 \pm 305^{*}$ & \\
EDPVR (/mL) & $8.6 \pm 2.9$ & $16.1 \pm 4.5^{*} \dagger$ & \\
PRSW (mm Hg) & $17.0 \pm 4.1$ & $40.2 \pm 19.6^{*}$ & \\
PRSW/RV (mm Hg/kg) & $88.3 \pm 23.9$ & $71.5 \pm 31.3$ & \\
\hline
\end{tabular}

$M S T$, Myoblast sheet transplantation; $H R$, heart rate; $E S P$, end-systolic pressure; $E D P$, end-diastolic pressure; $P V$, pressure-volume; $E S P V R$, end-systolic pressure-volume relationship; $E D P V R$, end-diastolic pressure-volume relationship; $P R S W$, preload-recruitable stroke work; $R V$, right ventricular weight. $* P<.05$ versus the control group. $\dagger P$ $<.05$ versus the MST group.

Factor VIII stain showed that myocardial capillary vascular density showed no significant difference at the mid layer and endocardial layer (Figure 3, $B$ and $C$ ), but it was significantly higher in the MST group than in the other 2 groups at the epicardial layer (Figure 3, A). Hence, total capillary density in the MST group was significantly higher than in the other 2 groups (control vs sham vs MST groups: $262 \pm 98$ vs $271 \pm 289$ vs $823 \pm 708 ; P<0.05$ in the control and sham groups vs the MST group; Figure 3, $D$ ).

Periodic acid-Schiff staining demonstrated significant interstitial fibrosis of the right ventricle in both the sham and MST groups, but the percentage of fibrosis in the control
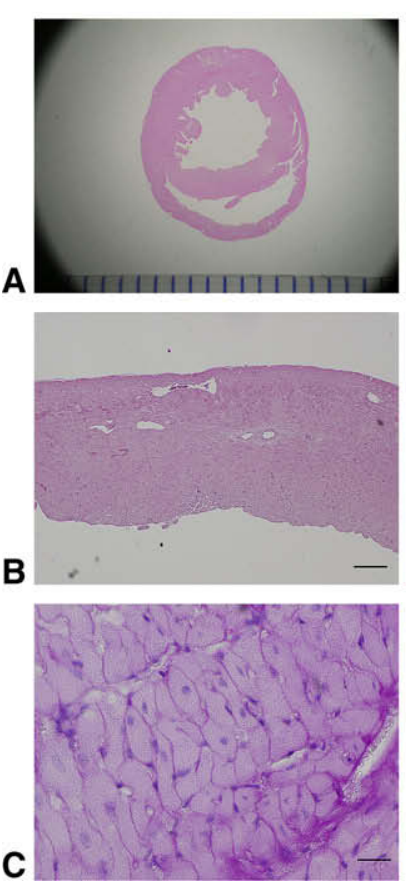

sham
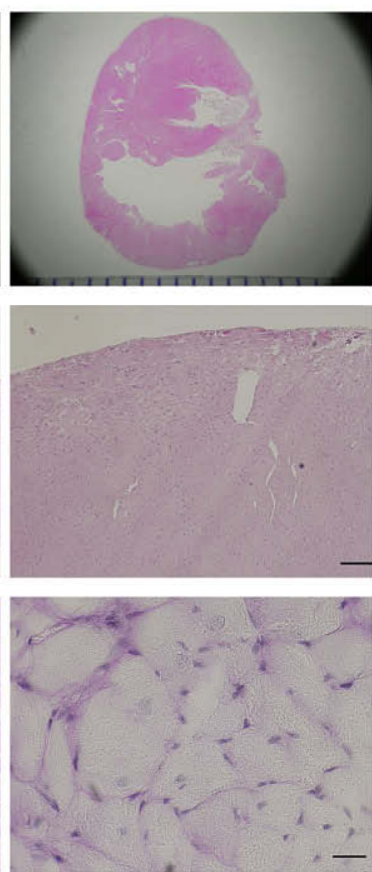

MST
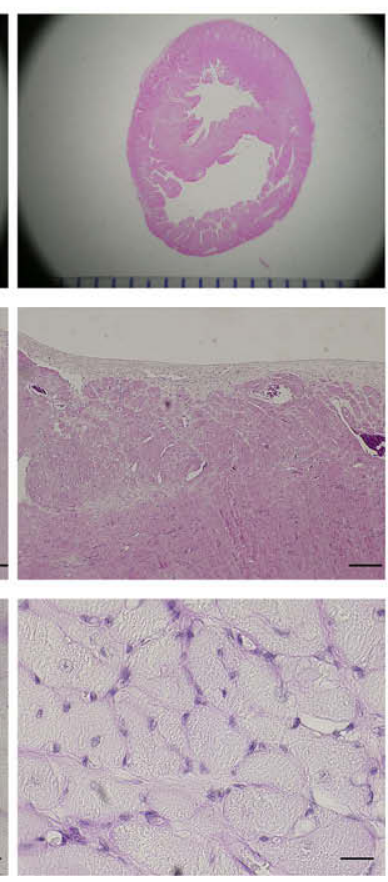

RV wall

thickness

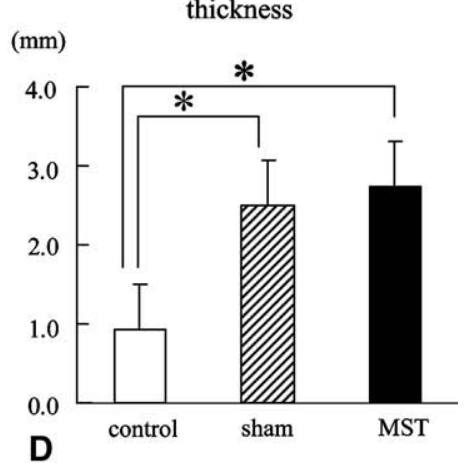

Cell size

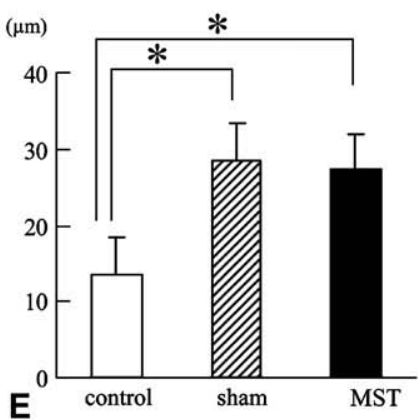

FIGURE 2. Macroscopic photographs of hematoxylin and eosin-stained sections showing right ventricular $(R V)$ wall thickening, cavity enlarging, and the intraventricular septum shifting towered the left side in the sham and myoblast sheet transplantation (MST) groups (A and D). Photomicrographs (40×, scale $\mathrm{bar}=200 \mu \mathrm{m}$ ) of hematoxylin and eosin-stained sections showed a fibrous organized thin layer on the epicardium in the myoblast sheet transplantation group (B). Photomicrographs $(400 \times$, scale bar $=20 \mu \mathrm{m})$ of periodic acid-Schiff-stained sections showed significantly hypertrophied ventricular myocytes in the sham and myoblast sheet transplantation groups $(\mathrm{C}$ and $\mathrm{E}) . * P<.05(\mathrm{n}=10)$. 


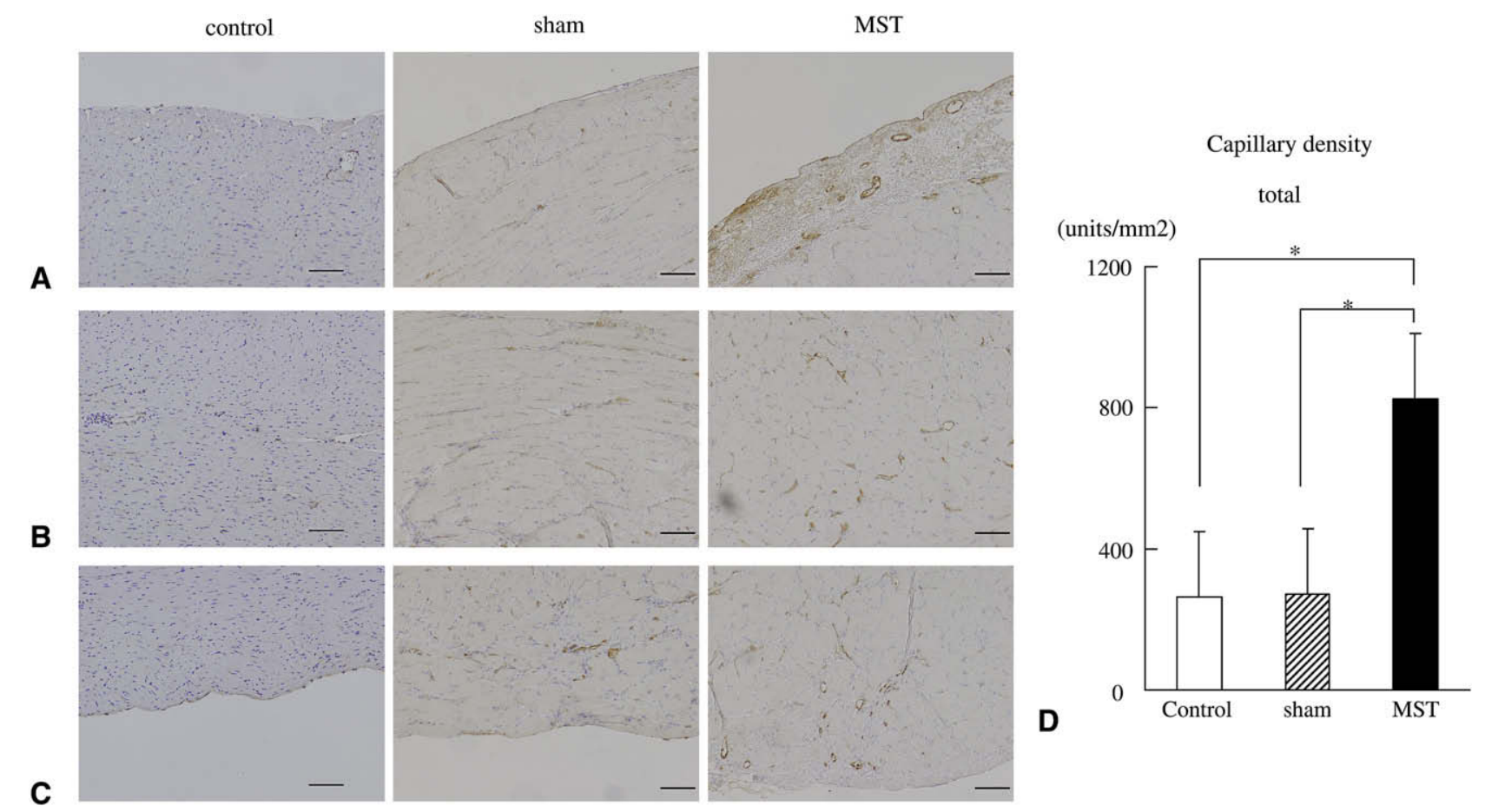

FIGURE 3. Representative photomicrographs $(100 \times$, scale bar $=100 \mu \mathrm{m})$ of Factor VIII-stained epicardial layer (A), mid layer (B), and endocardial layer (C). Neovascularization occurred at the epicardial layer in the myoblast sheet transplantation $(M S T)$ group (D). $* P<.05(\mathrm{n}=10)$.

MST group was significantly less than that in the sham group (control vs sham vs MST groups: $4.8 \% \pm 1.1 \%$ vs $24.5 \% \pm 10.0 \%$ vs $19.0 \% \pm 5.1 \% ; P<.05$ between each 2 groups; Figure 4, $A$ and $E$ ). Aggregated endomyocardial fibrosis was detected only in the sham group (endomyocardial percentage of fibrosis, control vs sham vs MST groups: $5.7 \% \pm 0.1 \%$ vs $31.5 \% \pm 8.4 \%$ vs $18.6 \% \pm$ $5.9 \% ; P<.01$ in the control and MST groups vs the sham group; Figure $4, B-D$ and $F$ ).

\section{RT-PCR}

The expression of HGF and VEGF mRNA in the MST group was significantly higher than in the sham group (control vs sham vs MST groups: HGF, $0.00009 \pm 0.00008$ vs $0.00041 \pm 0.00030$ vs $0.00073 \pm 0.00031 /$ GAPDH $[P<$ .05 in each group]; VEGF, $0.00242 \pm 0.00164$ vs 0.00329 \pm 0.00181 vs $0.00512 \pm 0.00113 /$ GAPDH $[P<.05$ in the control and sham group vs the MST group]; Figure 5).

\section{DISCUSSION}

This study demonstrated that skeletal MST improved diastolic function in a pressure-overloaded right heart model in rats by means of PAB. This conclusion is supported by the following evidence: (1) the diastolic function was significantly improved based on hemodynamic assessment and pressure-volume loop analysis; (2) interstitial and endocardial fibrosis was ameliorated, and capillary vascular density of the epicardial layer was increased; and (3) myocardial gene expression of HGF and VEGF was significantly increased. MST has been shown to have therapeutic effects in several models of LV failure. ${ }^{14-16}$ However, the present results are the first to show evidence that MST is effective for the treatment of RV dysfunction resulting from chronic pressure overload.

Prolonged RV pressure overload promotes unique morphologic, histologic, and functional changes. The mechanical stimulation of pressure overload extends the myocardium, which leads to diastolic dysfunction. ${ }^{21}$ Simultaneously, hypertrophied myocardium upregulates the release of various chemical mediators, ${ }^{17}$ which induce further myocardial expansion, apoptosis, necrosis, and fibrosis, finally resulting in $\mathrm{RV}$ decompensation. Otherwise, ventricular hypertrophy itself reduces the coronary flow reserve (CFR) and leads to coronary microcirculatory dysfunction. ${ }^{25}$ As studies on the left ventricle show, this phenomenon is detected particularly in the subendocardium. ${ }^{26}$ The shortage of CFR induces myocardial cellar mortality and endocardial fibrosis, which accelerates ventricular dysfunction. The present and previous data indicate that transplanted elastic cells initially improved ventricular stiffness ${ }^{14}$ and preserved CFR in the hypertrophied myocardium, both of which generate a synergistic effect of the suppression of myocardial cell death and fibrosis, especially at the endocardial layer. Although an angiogenetic effect was observed with the increased myocardial gene expression of HGF and VEGF, we speculate this does not increase endocardial coronary flow directly because increased 

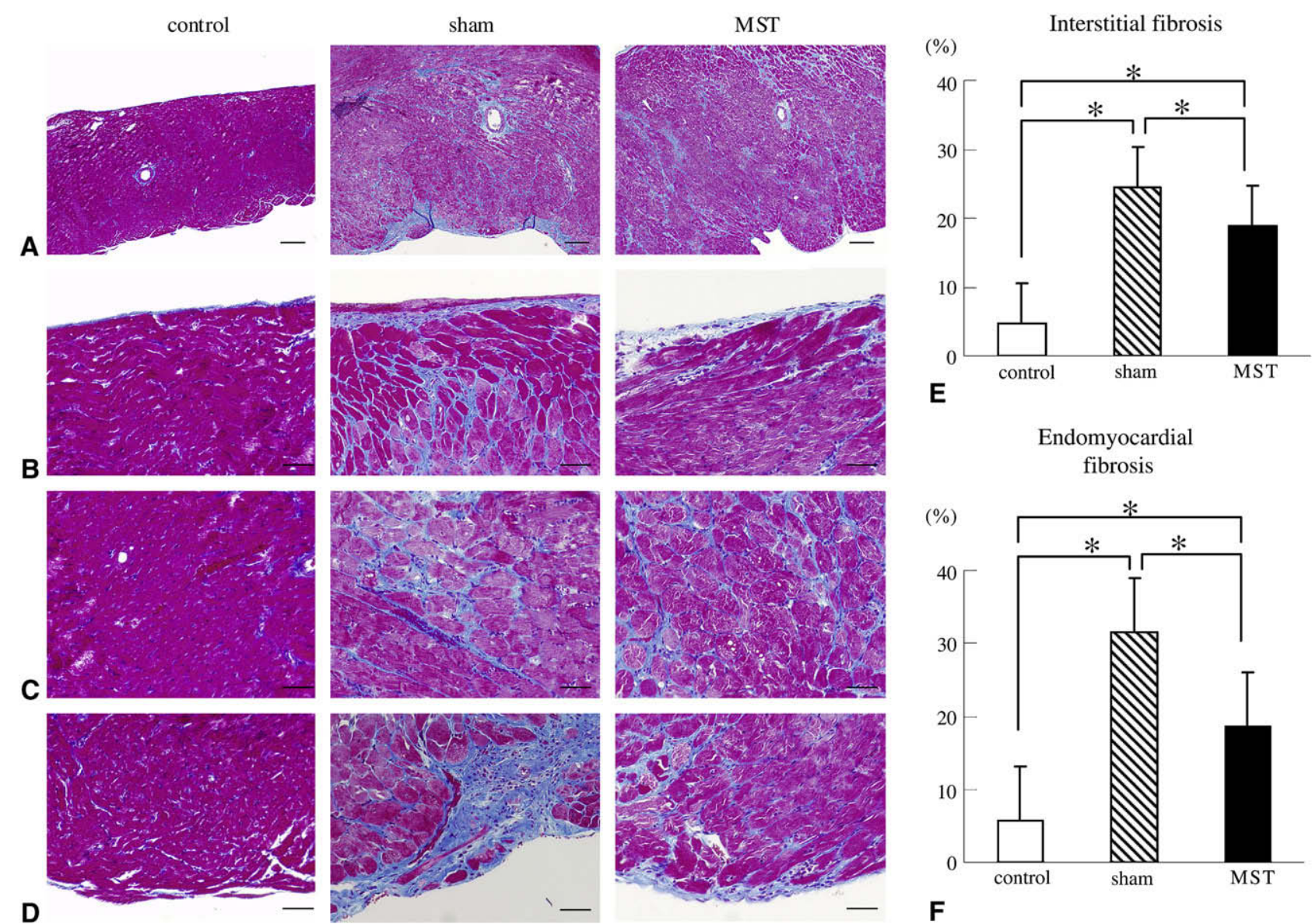

FIGURE 4. Representative photomicrographs of Masson's trichrome-stained transmural layer (A; 40×, scale bar = $200 \mu \mathrm{m}$ ), epicardial layer (B), mid layer $(\mathrm{C})$, and endocardial layer $(\mathrm{D} ; 200 \times$, scale bar $=50 \mu \mathrm{m})$ for evaluation of interstitial $(\mathrm{E})$ and endomyocardial $(\mathrm{F})$ fibrosis. $* P<.05(\mathrm{n}=10)$.

capillary density was only seen at epicardial layer. These new capillaries might increase the blood supply to the transplanted myoblasts and prolong their survival. ${ }^{13}$

As several LV studies have previously shown, Lamberts and colleagues ${ }^{27}$ revealed the strict relationship between RV chamber stiffness and the degree of myocardial fibrosis. In their report they also described that prevention or reduction of RV fibrosis improved RV diastolic dysfunction. Although we could not show any individual correlation between collagen contents and hemodynamic parameters in regard to diastolic function, these findings strongly support our results. Therefore we would like to emphasize that the suppression of fibrosis improved the RV diastolic function.

The hemodynamic assessment of cardiac performance of a hypertrophied right ventricle has not been established. Various attempts have been made; however, it is still necessary to perform cardiac catheterization to evaluate hemodynamics, especially regarding diastolic function. ${ }^{28,29}$ Leeuwenburgh and associates ${ }^{4}$ showed that RV compliance deteriorated in a lamb model and described $\tau$ and EDPVR values to be useful indicators for diastolic function. Gaynor
HGF

(/GAPDH)

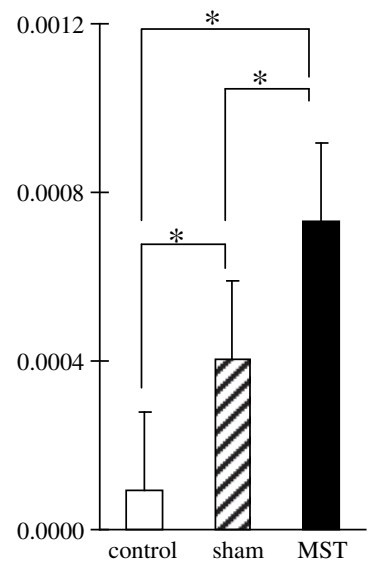

VEGF

(/GAPDH)

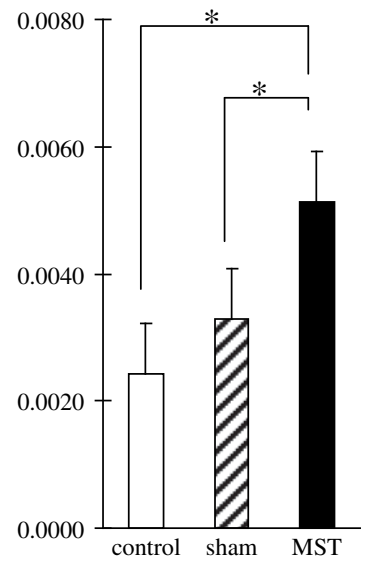

FIGURE 5. RT-PCR for evaluation of neurohormonal factor gene expression. The results are expressed after normalization for GAPDH. $H G F$, Hepatocyte growth factor; $V E G F$, vascular endothelial growth factor. $* P<.05(\mathrm{n}=10)$. 
and coworkers ${ }^{5}$ also showed an increase of EDPVR in a dog model of PAB. The current results are consistent with these reports. Therefore these data appear to be reliable.

On the other hand, the assessment of systolic function still remains controversial. In this study we showed that ESPVR (Ees) and PRSW were significantly increased in sham groups, as previously reported. ${ }^{30}$ However, Dell'Italia and Walsh ${ }^{31}$ pointed out that in the assessment of RV contractility, the slope of ESPVR (Ees) was different from the slope of the maximum time-varying elastance, which better reflected the RV contractility than Ees. In addition to Ees, PRSW is thought to be an optimal parameter of ventricular contractility. However, we might have to consider the discrepancy of the cardiac mass between normal and hypertrophied hearts because ventricular SW should be assessed as a per-unit cardiac mass. We tried to calculate the PRSW divided for each animal's RV weight, which revealed there was no statistical significance among the 3 groups (control vs sham vs MST groups: $88.3 \pm 23.9$ vs $71.5 \pm 31.3$ vs $73.6 \pm 28.8 \mathrm{~mm}$ $\mathrm{Hg} / \mathrm{kg}$, Table 2). Hence it is hard to say that the RV pressure load induced a further improvement in RV contractility.

We need further investigation to apply this method to clinical RV failure because we did not ascertain the effect of MST on RV systolic function. Nevertheless, enormous fibrosis was seen in the sham group, and systolic function was still compensated until the timing of hemodynamic evaluation. However, previous reports showed that MST had an excellent effect on LV contractility. ${ }^{14-16}$ Therefore we expect that this method might become a novel and potentially effective treatment strategy for patients with RV failure.

In conclusion, chronic pressure overload to the right ventricle caused hypertrophy and impaired diastolic function in rats. Skeletal MST attenuated diastolic dysfunction, which was mainly caused by suppressed interstitial and endocardial fibrosis. This method might become a novel strategy for the myocardial regeneration of $\mathrm{RV}$ failure in patients with $\mathrm{CHD}$ in the future.

We thank Mrs Masako Yokoyama for her expert assistance with RT-PCR and Kazuhiro Takekita and Takeshi Miki for technical support in creating the tissue-engineered skeletal MSs.

\section{References}

1. Bolger AP, Coats AJ, Gatzoulis MA. Congenital heart disease: the original heart failure syndrome. Eur Heart J. 2003;24:970-6.

2. Murphy JG, Gersh BJ, Mair DD, Fuster V, McGoon MD, Ilstrup DM, et al. Longterm outcome in patients undergoing surgical repair of tetralogy of Fallot. $N$ Engl J Med. 1993;329:593-9.

3. Gatzoulis MA, Balaji S, Webber SA, Siu SC, Hokanson JS, Poile C, et al. Risk factors for arrhythmia and sudden cardiac death late after repair of tetralogy of Fallot: a multicentre study. Lancet. 2000;356:975-81.

4. Leeuwenburgh BP, Steendijk P, Helbing WA, Baan J. Indexes of diastolic RV function: load dependence and changes after chronic RV pressure overload in lambs. Am J Physiol Heart Circ Physiol. 2002;282:1350-8.

5. Gaynor SL, Maniar HS, Bloch JB, Steendijk P, Moon MR. Right atrial and ventricular adaptation to chronic right ventricular pressure overload. Circulation. 2005;112:212-8.
6. Deanfield JE, Ho SY, Anderson RH, McKenna WJ, Allwork SP, HallidieSmith KA. Late sudden death after repair of tetralogy of Fallot: a clinicopathologic study. Circulation. 1983;67:626-31.

7. Babu-Narayan SV, Kilner PJ, Li W, Moon JC, Goktekin O, Davlouros PA, et al. Ventricular fibrosis suggested by cardiovascular magnetic resonance in adults with repaired tetralogy of Fallot and its relationship to adverse markers of clinical outcome. Circulation. 2006;113:405-13.

8. Chowdhury UK, Sathia S, Ray R, Singh R, Pradeep KK, Venugopal P. Histopathology of the right ventricular outflow tract and its relationship to clinical outcomes and arrhythmias in patients with tetralogy of Fallot. $J$ Thorac Cardiovasc Surg. 2006;132:270-7.

9. Taylor DA, Atkins BZ, Hungspreugs P, Jones TR, Reedy MC, Hutcheson KA, et al. Regenerating functional myocardium: improved performance after skeletal myoblast transplantation. Nat Med. 1998;4:929-33.

10. Menasche P, Hagege AA, Scorsin M, Pouzet B, Desnos M, Duboc D, et al. Myoblast transplantation for heart failure. Lancet. 2001;357:279-80.

11. Hagege AA, Marolleau JP, Vilquin JT, Alheritiere A, Peyrard S, Duboc D, et al. Skeletal myoblast transplantation in ischemic heart failure: long-term follow-up of the first phase I cohort of patients. Circulation. 2006;114:108-13.

12. Okano T, Yamada N, Sakai H, Sakurai Y. A novel recovery system for cultured cells using plasma-treated polystyrene dishes grafted with poly( $\mathrm{N}$-isopropylacrylamide). J Biomed Mater Res. 1993;27:1243-51.

13. Miyagawa S, Sawa Y, Sakakida S, Taketani S, Kondoh H, Memon IA, et al. Tissue cardiomyoplasty using bioengineered contractile cardiomyocyte sheets to repair damaged myocardium: their integration with recipient myocardium. Transplantation. 2005;80:1586-95.

14. Memon IA, Sawa Y, Fukushima N, Matsumiya G, Miyagawa S, Taketani S, et al. Repair of impaired myocardium by means of implantation of engineered autologous myoblast sheets. J Thorac Cardiovasc Surg. 2005;130:1333-41.

15. Kondoh H, Sawa Y, Miyagawa S, Sakakida-Kitagawa S, Memon IA, Kawaguchi N, et al. Longer preservation of cardiac performance by sheet-shaped myoblast implantation in dilated cardiomyopathic hamsters. Cardiovasc Res. 2006;69:466-75.

16. Hata H, Matsumiya G, Miyagawa S, Kondoh H, Kawaguchi N, Matsuura N, et al. Grafted skeletal myoblast sheets attenuate myocardial remodeling in pacing-induced canine heart failure model. J Thorac Cardiovasc Surg. 2006;132: 918-24.

17. Lekanne Deprez RH, van den Hoff MJ, de Boer PA, Ruijter PM, Maas AA, Chamuleau RA, et al. Changing patterns of gene expression in the pulmonary trunk-banded rat heart. J Mol Cell Cardiol. 1998;30:1877-88.

18. Sato T, Shishido T, Kawada T, Miyano H, Miyashita H, Inagaki M, et al. ESPVR of in situ rat left ventricle shows contractility-dependent curvilinearity. Am J Physiol Heart Circ Physiol. 1998;274:H1429-34.

19. Baan J, van der Velde ET, de Bruin HG, Smeenk GJ, Koops J, van Dijk AD, et al. Continuous measurement of left ventricular volume in animals and humans by conductance catheter. Circulation. 1984;70:812-23.

20. Suga $\mathrm{H}$, Sagawa K. Instantaneous pressure-volume relationships and their ratio in the excised, supported canine left ventricle. Circ Res. 1974;35:117-26.

21. Gaasch WH, Cole JS, Quinones MA, Alexander JK. Dynamic determinants of left ventricular diastolic pressure-volume relations in man. Circulation. 1975;51: 317-23.

22. Glower DD, Spratt JA, Snow ND, Kabas JS, Davis JW, Olsen CO, et al. Linearity of the Frank-Starling relationship in the intact heart: the concept of preload recruitable stroke work. Circulation. 1985;71:994-1009.

23. Hoyt RH, Collins SM, Skorton DJ, Ericksen EE, Conyers D. Assessment of fibrosis in infarcted human hearts by analysis of ultrasonic backscatter. Circulation. 1985;71:740-4.

24. Vasiljević JD, Popović ZB, Otasević P, Popović ZV, Vidaković R, Mirić M, et al. Myocardial fibrosis assessment by semiquantitative, point-counting and computer-based methods in patients with heart muscle disease: a comparative study. Histopathology. 2001;38:338-43.

25. Murray PA, Vatner SF. Reduction of maximal coronary vasodilator capacity in conscious dogs with severe right ventricular hypertrophy. Circ Res. 1981;48: 25-33.

26. Rajappan K, Rimoldi OE, Dutka DP, Ariff B, Pennell DJ, Sheridan DJ, et al. Mechanisms of coronary microcirculatory dysfunction in patients with aortic stenosis and angiographically normal coronary arteries. Circulation. 2002;105:470-6.

27. Lamberts RR, Caldenhoven E, Lansink M, Witte G, Vaessen RJ, St Cyr JA, et al. Preservation of diastolic function in monocrotaline-induced right ventricular hypertrophy in rats. Am J Physiol Heart Circ Physiol. 2007;293: H1869-76. 
28. Haddad F, Hunt SA, Rosenthal DN, Murphy DJ. Right ventricular function in cardiovascular disease, part I: anatomy, physiology, aging, and functional assessment of the right ventricle. Circulation. 2008;117:1436-48.

29. Burkhoff D, Mirsky I, Suga H. Assessment of systolic and diastolic ventricular properties via pressure-volume analysis: a guide for clinical, translational, and basic researchers. Am J Physiol Heart Circ Physiol. 2005;289:H501-12.
30. Faber MJ, Dalinghaus M, Lankhuizen IM, Steendijk P, Hop WC, Schoemaker RG, et al. Right and left ventricular function after chronic pulmonary artery banding in rats assessed with biventricular pressure-volume loops. Am J Physiol Heart Circ Physiol. 2006;291:H1580-6.

31. Dell'Italia LJ, Walsh RA. Application of a time varying elastance model to right ventricular performance in man. Cardiovasc Res. 1988;22:864-74. 